$(\mathrm{p}<0.01)$ (figure 1). Pearson correlation coefficient showed positive linear correlation between fluorescence values and histological steatosis grading $(\mathrm{r}=0.4337 ; \mathrm{p}<0.05)$. Greater fluorescence in steatotic hepatocytes was observed under fluorescence microscope due to high lipid content compared to non-steatotic cells (figure 2).

Summary and Conclusion Our study shows that Nile Red staining was an easy, quick, and reliable technique for grading steatotic hepatocytes isolated from a liver biopsy of a patient with NAFLD or NASH, or from a donor liver to be used in transplantation. These results can be combined with a FibroScan to give a useful quantitative measure of steatosis and fibrosis in a sample of hepatocytes.

\section{P60 USTEKINUMAB IS AN EFFECTIVE DRUG FOR STEROID- FREE REMISSION IN CHILDREN WITH REFRACTORY IBD AND ANTI TNF-ALPHA INDUCED PSORIASIS}

Farah M Barakat, Claire Barnes, Jonathan Baker, Akshay Batra, Nadeem A Afzal, R Mark Beattie, Tracy A Coelho. Southampton General Hospital

\subsection{6/flgastro-2021-bspghan.69}

Background Management of paediatric Crohn's disease (CD) presents significant challenges. Escalation of therapy to biologics or a 'top-down' approach, with early introduction of biologics, is common. Anti-TNF agents are widely used, but the use of ustekinumab is still limited. Ustekinumab is a human monoclonal antibody targeting the p40 subunit of both IL-12/-23. Ustekinumab has recently emerged as an alternative treatment option in children with failure of response to anti-TNF alpha treatment and those developing intolerable side effects on anti-TNF treatment including psoriasis.

Aims Data on ustekinumab efficacy in paediatric IBD are limited. We report our experience on the use of this monoclonal antibody in paediatric IBD at a tertiary GI service in Wessex. Methods Retrospective review of all paediatric patients $(\leq 18$ years) receiving ustekinumab for the management of their IBD. Data was collected by reviewing patients' electronic medical records and available results. Only those who had the treatment for at least 26 weeks were included. Steroid free remission were the primary outcomes of this study and resolution of psoriasis (where applicable) as a secondary outcome measure.

Results Between November 2017- November 2020, 12 patients $(\mathrm{M}: \mathrm{F}=6: 6)$ patients were identified who were commenced on ustekinumab for the treatment of their IBD; 9 patients with CD and 3 with IBDU- Crohn's like. One patient was excluded as the duration of treatment was $<26$ weeks at the time of the review. The median age at diagnosis was 11 years and median age for initiating treatment with ustekinumab was 13 years (11-17 years).

The median duration between diagnosis and initiation of treatment with ustekinumab was 104 weeks and median duration of treatment with ustekinumab at the time of the review was 60 weeks (26-160 weeks).

All patients received standard anti-TNF alpha treatment prior to ustekinumab. (All 11 patients received infliximab, 7 patients received both infliximab and adalimumab, and 4 patients were escalated directly from infliximab to ustekinumab). The key indications for considering ustekinumab were primary non-response $(\mathrm{N}=5,45 \%)$, secondary loss of response $(\mathrm{N}=3,27 \%)$ and anti-TNF-alpha induced psoriasis. Six patients (54\%) developed psoriasis while on treatment with anti-TNF alpha. Three patients $(27 \%)$ were switched to ustekinumab primarily in view of anti-TNF-alpha induced psoriasis.

Steroid-free remission rates were (81\%) at 26 weeks $(\mathrm{N}=9),(90 \%)$ at 52 weeks and $100 \%$ thereafter in 5 individuals who remained on ustekinumab over 1 year at the last review. No significant side-effects were reported in any patient.

$85 \%$ of patients $(\mathrm{N}=5: 6)$ who were identified with psoriasis have shown good response to switching treatment from anti-TNF to ustekinumab.

Conclusions This data suggests that ustekinumab is a useful and a safe treatment option in children with IBD refractory to standard monoclonal therapy and in those developing intolerable anti-TNF alpha induced side-effects, psoriasis in particular. Larger studies from multiple centres would be required to develop standardised pathways for the use of this promising monoclonal agent for the management of paediatric IBD. 